Publ. Mat. 58 (2014), 469-484

DOI: 10.5565 /PUBLMAT_58214_23

\title{
THIRD-POWER ASSOCIATIVE ABSOLUTE VALUED ALGEBRAS WITH A NONZERO IDEMPOTENT COMMUTING WITH ALL IDEMPOTENTS
}

\author{
José Antonio Cuenca Mira
}

\begin{abstract}
This paper deals with the determination of the absolute valued algebras with a nonzero idempotent commuting with the remaining idempotents and satisfying $x^{2} x=x x^{2}$ for every $x$. We prove that, in addition to the absolute valued algebras $\mathbb{R}$, $\mathbb{C}, \mathbb{H}$, or $\mathbb{O}$ of the reals, complexes, division real quaternions or division real octonions, one such absolute valued algebra $A$ can also be isometrically isomorphic to some of the absolute valued algebras $\stackrel{\star}{\mathbb{C}}, \stackrel{\star}{\mathbb{H}}$, or $\stackrel{\star}{\mathbb{O}}$, obtained from $\mathbb{C}, \mathbb{H}$, and $\mathbb{O}$ by imposing a new product defined by multiplying the conjugates of the elements. In particular, every absolute valued algebra having the above properties is finite-dimensional. This generalizes some well known theorems of Albert, Urbanik and Wright, and El-Mallah.
\end{abstract}

2010 Mathematics Subject Classification: 17A80, 17A75, 17A60, 17D99.

Key words: Absolute valued algebra, idempotent, division algebra, third-power associativity, pairwise commuting elements.

\section{Introduction}

Let $A \neq 0$ be a real algebra, which is not necessarily associative as all the algebras considered throughout this note. The algebra $A$ is said to be an absolute valued algebra if its vector space is a real normed space and

$$
\|x y\|=\|x\|\|y\|
$$

holds for any $x, y \in A$. As an immediate consequence from (1) we obtain that the linear operators $L_{x}, R_{x}: A \longrightarrow A$ given by $L_{x}: y \mapsto x y$ and $R_{x}: y \mapsto y x$ are one to one maps for any $x \neq 0$. Real and complex numbers, so as the division quaternions and division octonions algebras $\mathbb{H}$ and $\mathbb{O}$ are absolute valued algebras. A celebrated theorem by $\mathrm{K}$. Urbanik and F. B. Wright asserts that $\mathbb{R}, \mathbb{C}, \mathbb{H}$, and $\mathbb{O}$ are the only unital absolute valued algebras [16, Theorem 1]. According to [14, p. 109], we will call it the noncommutative Urbanik and Wright Theorem. This

This work was partially supported by MINECO (MTM2010-18370-C04-04). 
was previously stated by A. A. Albert under the additional restriction of algebraicity [2].

This paper deals with absolute valued algebras that are third-power associative. We recall that an algebra $A$ over a field $F$ is said to be third-power associative, or that $A$ satisfies the third-power associative condition, if $x^{2} x=x x^{2}$ for all elements $x$ of $A$. It is possible to obtain easily from $\mathbb{R}, \mathbb{C}, \mathbb{H}$, or $\mathbb{O}$ examples of third-power associative absolute valued algebras in the following way: if $D=\mathbb{C}, \mathbb{H}$, or $\mathbb{O}$ and $\star$ is its natural involution then the algebra $\stackrel{\star}{D}$ obtained from $D$ by changing the product into the new product - defined by $x \cdot y=x^{\star} y^{\star}$ is an absolute valued algebra with respect to the same norm as that of $D$. The absolute valued algebras $\stackrel{\star}{\mathbb{C}}, \stackrel{\star}{\mathbb{H}}, \stackrel{\star}{\mathbb{O}}$ are third-power associative. The first of these, $\stackrel{\star}{\mathbb{C}}$, has been previously considered by many authors including Albert [3] and Urbanik and Wright [16]. In fact in the fundamental paper $[\mathbf{1 6}]$ the authors prove that, up to isometric isomorphisms, $\mathbb{R}, \mathbb{C}$, and $\stackrel{\star}{\mathbb{C}}$ are the unique commutative absolute valued algebras. According to [14] we will call this result the commutative Urbanik and Wright Theorem.

The norm of the absolute valued algebras $\stackrel{\star}{\mathbb{C}}, \stackrel{\star}{\mathbb{H}}, \stackrel{\star}{\mathbb{O}}$ derives from an inner product. Furthermore, they have nonzero idempotents, but they are not unital. Nevertheless, the three algebras have a nonzero idempotent $e$ satisfying

$$
e x=-x=x e
$$

for every element $x$ orthogonal to $e$. A nonzero idempotent in an absolute valued algebra $A$ with norm deriving from an inner product is said to be a para-unit if (2) holds for every $x$ orthogonal to $e$. Obviously each para-unit is a nonzero idempotent commuting with each element of $A$. In particular, it commutes with all idempotents.

The above comments suggest the natural question: whether in a given third-power associative absolute valued algebra $A$ the existence of a nonzero idempotent commuting with all idempotents guarantees that $A$ is isometrically isomorphic to $\mathbb{R}, \mathbb{C}, \mathbb{H}, \mathbb{O}, \stackrel{\star}{\mathbb{C}}, \stackrel{\star}{\mathbb{H}}$, or $\stackrel{\star}{\mathbb{O}}$. The main theorem in this paper gives an affirmative answer to this problem. Neither the third-power associativity nor the existence of a nonzero idempotent commuting with the remaining can be dropped in the assumptions of our main theorem. Indeed, it is well-known that infinite-dimensional absolute valued algebras with a unique nonzero idempotent exist even satisfying, in addition, to be a one-sided division algebra (see $[\mathbf{6}, \mathbf{1 3}]$ ). As a consequence of our main theorem, we can obtain the commutative 
Urbanik and Wright Theorem, as well as a result by A. Chandid asserting that the nonzero idempotents in a third-power associative absolute valued algebra $A$ commute pairwise if and only if $A$ is isometrically isomorphic to $\mathbb{R}, \mathbb{C}, \mathbb{H}, \mathbb{O}$, or $\stackrel{\star}{\mathbb{C}}$ (see [5, Théorème 3.19]).

In order to prove our main theorem we need to establish some results, sometimes related to the commutativity of certain elements in the absolute valued algebra. In general, Section 2 is devoted to those results that can be proved without the requirement of the third-power associativity. This includes Proposition 2.5 which assures that certain subspaces of elements commuting with a given nonzero idempotent have norm deriving from an inner product. In Section 3 we study some aspects associated with the nonzero idempotents in a third-power associative absolute valued algebra. The existence of these idempotents is a consequence of a very recent result established by M. Cabrera and A. Rodríguez in the forthcoming book [4], and formulated in Proposition 3.1 below. We will use it in the proof of the main theorem. Also Proposition 3.5 depends of it. We continue Section 3 by giving several properties related to idempotents satisfying some restrictions on commutativity (Propositions 3.2, 3.4 , and 3.5). In the remainder of the section we focus on third-power associative absolute valued algebras $A$ with a nonzero idempotent $g$ commuting with all the idempotents of $A$ and we obtain a decomposition into vector subspaces of $A$ which plays a role similar to the Peirce decomposition in classical contexts, establishing, in addition, some properties of these subspaces (Proposition 3.6). As application, we give a new proof of a theorem by M. L. El-Mallah, which asserts that every third-power associative absolute valued algebra $A$ with a nonzero idempotent commuting with the remaining elements of $A$ is isometrically isomorphic to $\mathbb{R}, \mathbb{C}, \mathbb{H}, \mathbb{O}, \stackrel{\star}{\mathbb{C}}, \stackrel{\star}{\mathbb{H}}$, or $\stackrel{\star}{\mathbb{O}}($ see $[\mathbf{1 0}$, Theorem 3.7] and $[\mathbf{9}$, Theorem 6$]$ ).

Finally we point out that despite all efforts realized and the partial successes, the problem of the determination of all the third-power associative absolute valued algebras still remains open (see the excellent survey [14] for a more complete information). This makes that the results obtained and the methods developed throughout this paper can also be viewed as a new contribution to this topic.

\section{Commuting elements in absolute valued algebras}

Let $A$ be an absolute valued algebra and $W$ a vector subspace of $A$ whose elements pairwise commute. It is well known that the norm in the vector subspace $W$ derives from an inner product $(\mid)_{W}$ (see $[\mathbf{1 6}$, Lemma 1]). If $x$ and $y$ commute in $A$ we will denote by $(\mid)_{x, y}$ the inner 
product inducing the norm in $\mathbb{R} x+\mathbb{R} y$. For an absolute valued algebra $A$ satisfying the third-power associative condition the elements of everyone of the subspaces $\mathbb{R} x+\mathbb{R} x^{2}$ pairwise commute. For short, we will write then the inner products of these subspaces as $(\mid)_{x, 2}$.

Lemma 2.1. Let $x$ and $y$ be commuting elements in an absolute valued algebra $A$ such that the squares $x^{2}$ and $y^{2}$ also commute.

1. If $\|x\|=1=\|y\|$ and $(x \mid y)_{x, y}=0$ then $x^{2}=-y^{2}$.

2. If $x^{2}$ commutes with $x y$, then

$$
\|x\|^{2} y^{2}-2(x \mid y)_{x, y} x y+\|y\|^{2} x^{2}=0 .
$$

In particular, if $e$ and $f$ are different nonzero idempotents such that $\mathbb{R} e+\mathbb{R} f$ is a commutative subalgebra then this subalgebra is isometrically isomorphic to $\stackrel{\star}{\mathbb{C}}$.

Proof: 1 . We have $x^{2}-y^{2}=(x+y)(x-y)$ and so $\left\|x^{2}-y^{2}\right\|^{2}=\| x+$ $y\left\|^{2}\right\| x-y \|^{2}=\left(\|x\|^{2}+\|y\|^{2}\right)^{2}=4$. Thus, $\left\|x^{2}-y^{2}\right\|=2$. By $[\mathbf{1 6}$, Lemma 3], we obtain $x^{2}=-y^{2}$.

2. If $x$ and $y$ belong to the same vector line, (3) holds trivially. We will assume $x$ and $y$ linearly independent. If $x$ and $y$ are orthogonal, applying part 1 to $x /\|x\|$ and $y /\|y\|$, we obtain $\|y\|^{2} x^{2}+\|x\|^{2} y^{2}=0$, which is just (3). Now we will consider the case that $x$ and $y$ are linearly independent but not necessarily orthogonal. A straightforward verification shows that $x^{\prime}:=x$ and $y^{\prime}:=-(x \mid y)_{x, y} x+\|x\|^{2} y$ are linearly independent commuting orthogonal elements whose squares also commute. The previous case when the elements are orthogonal yields to

$$
\left\|y^{\prime}\right\|^{2} x^{\prime 2}+\left\|x^{\prime}\right\|^{2} y^{\prime 2}=0 .
$$

Since $y^{\prime 2}=(x \mid y)_{x, y}^{2} x^{2}-2(x \mid y)_{x, y}\|x\|^{2} x y+\|x\|^{4} y^{2}$ and $\left\|y^{\prime}\right\|^{2}=$ $\|x\|^{4}\|y\|^{2}-(x \mid y)_{x, y}^{2}\|x\|^{2}$, the relation (4) remains

$$
\|x\|^{4}\|y\|^{2} x^{2}-2(x \mid y)_{x, y}\|x\|^{4} x y+\|x\|^{6} y^{2}=0,
$$

which is equivalent to $(3)$.

In the case that $e$ and $f$ are different nonzero idempotents such that $\mathbb{R} e+\mathbb{R} f$ is a commutative subalgebra, the specialization of (3) to $x=e$ and $y=f$ gives $e+f=2(e \mid f)_{e, f}$ ef and in consequence $4(e \mid f)^{2}=$ $\|e+f\|^{2}=2+2(e \mid f)$. The solutions of the second degree equation $2(e \mid$ $f)^{2}-(e \mid f)-1=0$ are $(e \mid f)=1$ and $(e \mid f)=-\frac{1}{2}$, but the first possibility can be ruled out, because $(e \mid f)=1=\|e\|\|f\|$ is the equality case of the Cauchy-Schwartz inequality, so $e=f$, a contradiction. Thus, 
$(e \mid f)=-\frac{1}{2}$ and $e+f+e f=0$. Therefore, $\mathbb{R} e+\mathbb{R} f$ is isometrically isomorphic to $\stackrel{\star}{\mathbb{C}}$.

Remark 2.2. Part 1 of the previous lemma is well known in the particular case that one of the given commuting elements is an idempotent [10, Lemma 3.3] and it also appears implicitly in [16]. An identity as that of equation (3) holds in the related frame of the finite-dimensional (resp. anisotropic) third-power associative composition algebras, even without any assumption on commutativities involving squares [12] (see also [7] where this identity plays an important role in the determination of the third-power associative anisotropic composition algebras over fields of characteristic $\neq 2,3$ ). It also appears in [5, Proposition 3.21] in the context of the absolute valued algebras. The assertion in 2 relative to the subalgebra $\mathbb{R} e+\mathbb{R} f$ is a particular case of Albert's result asserting that finite-dimensional absolute valued algebras are isotopic to $\mathbb{R}$, $\mathbb{C}, \mathbb{H}$, or $\mathbb{O}[\mathbf{1}, \mathbf{3}]$, which implies that any two-dimensional commutative absolute valued algebra is isometrically isomorphic to $\mathbb{C}$ or $\stackrel{\star}{\mathbb{C}}$.

Corollary 2.3. Let $A$ be a third-power associative absolute valued algebra and $x \in A$ such that $\|x\|=1$ and $x^{2} x^{3}=x^{3} x^{2}$. Then

$$
x^{2} x^{2}-2\left(x \mid x^{2}\right)_{x, 2} x^{3}+x^{2}=0 .
$$

Remark 2.4. If $x$ and $y$ are commuting elements in an absolute valued algebra and $W$ is a prehilbert space containing $\mathbb{R} x+\mathbb{R} y$, then $(z \mid v)_{x, y}=$ $(z \mid v)_{W}$ for all $z, v \in \mathbb{R} x+\mathbb{R} y$ and so in it is possible to substitute the inner product of (3) (resp. (5)) by $(x \mid y)_{W}$ (resp. $\left.\left(x \mid x^{2}\right)_{W}\right)$. In particular, this is so when $W$ is a vector subspace of $A$ containing $x$ and $y$ and where its elements pairwise commute.

If $F$ is a field of characteristic $\neq 2$ and $A$ an $F$-algebra, as it is customary, we will denote by $\bullet$ the new product in $A$ defined as $x \bullet y=$ $\frac{1}{2}(x y+y x)$. In this way we obtain a new algebra $A^{+}$which is said to be the symmetrized algebra of $A$. Also • is called the symmetrized product of that of $A$.

The next proposition is an easy consequence of the results in [7].

Proposition 2.5. Let $A$ be an absolute valued algebra, $g \neq 0$ an idempotent, and $B$ a vector subspace containing $g$. Assume that every $x \in B$ generates with $g$ a subalgebra isomorphic to $\mathbb{R}, \mathbb{C}$, or $\stackrel{\star}{\mathbb{C}}$. Then $B$ is a subalgebra of $A^{+}$, the norm of $B$ derives from an inner product $(\mid)$ and if $V$ is the vector subspace of the elements orthogonal to $g$ in $B$ then the 
commutative symmetrized product $\bullet$ of $B$ is given in the way

$$
(\alpha g+v) \cdot(\beta g+w)=(\alpha \beta-(v \mid w)) g+\alpha g w+\beta g v
$$

for any $\alpha, \beta \in \mathbb{R}$ and $v, w \in V$. Furthermore, $g v= \pm v$ for any $v \in V$.

Proof: Since every element $x$ of $B$ generates with $g$ a subalgebra $B_{x}$ of $A$ isomorphic to $\mathbb{R}, \mathbb{C}$, or $\stackrel{\star}{\mathbb{C}}, g$ commutes with $x$ and $B_{x} \subset B$. Furthermore, $\left\|z \bullet z^{\prime}\right\|=\left\|z z^{\prime}\right\|=\|z\|\left\|z^{\prime}\right\|$ for all $z, z^{\prime} \in B_{x}$. Since $x^{2} \in B_{x} \subset B$, the vector subspace $B$ is closed by squares, so it is also a subalgebra of $A^{+}$. By [7, Proposition 3.7], $B$ with the product $\bullet$ is a commutative $g$-quadratic algebra in the sense of $[7]$ and this product is given as in (6) (see also [7, Lemma 3.1 and proof of Lemma 3.6]). Finally, if $v \in V$ then the orthogonality of $g$ and $v$ and the fact that $B_{v}$ is isometrically isomorphic to $\mathbb{C}$ or $\stackrel{\star}{\mathbb{C}}$ imply that $g v= \pm v$.

\section{Idempotents in third-power associative algebras}

This section deals with some properties associated with nonzero idempotents in third-power associative absolute valued algebras. We first consider the existence of these idempotents in one such absolute valued algebra $A$. If $x$ is a norm-one element of $A$ such that $x$ and $x^{2}$ are linearly dependent then $x^{2}= \pm x$, which gives that $x$ or $-x$ is a nonzero idempotent in $A$. In case that $x$ and $x^{2}$ are linearly independent, the existence of nonzero idempotents is guaranteed by the proposition below, which is proved in a forthcoming book by M. Cabrera and A. Rodríguez [4, Proposition 2.8.85]. Our proof of the main theorem of this paper depends also heavily on this proposition.

Proposition 3.1. Let $A$ be a third-power associative absolute valued algebra and $P$ a vector plane in $A$. Then there exists some nonzero element in the set $\left\{z \in P:\left(z \mid z^{2}\right)_{z, 2}=0\right\}$ and if $z$ is a norm-one element in this set then $-z^{2}$ is a nonzero idempotent of $A$.

Next we recall some elementary facts on third-power associative algebras. Let $A$ be a third-power associative algebra over a field of characteristic $\neq 2$. By linearization of $x x^{2}=x^{2} x$ it follows

$$
y x^{2}+x(x y+y x)=(x y+y x) x+x^{2} y .
$$

Using the bracket product $[$,$] defined by [x, y]=x y-y x$ for all $x, y \in A$, the identity (7) can be also written in the way

$$
[x y+y x, x]+\left[x^{2}, y\right]=0 .
$$


Making $y=x^{2}$ in the previous equation we obtain $x\left(x^{2} x\right)=\left(x^{2} x\right) x$. Let $e$ be an arbitrary idempotent in $A$. From (7) we get

$$
L_{e} \circ\left(L_{e}+R_{e}-\mathrm{Id}\right)=R_{e} \circ\left(L_{e}+R_{e}-\mathrm{Id}\right),
$$

which asserts that $e x+x e-x$ commutes with $e$ for every $x \in A$. Using the symmetrized product, the linearized identity (8) can be written in a more compact way as $2[x \bullet y, x]+\left[x^{2}, y\right]=0$.

There are some elementary properties related to the nonzero idempotents of an algebra, which can be useful. So if $A$ is an algebra over a field of characteristic $\neq 2$ and $e$ and $f$ nonzero idempotents of $A$ then $-e \neq f$. Indeed, in the contrary case $-e=f=f^{2}=e^{2}=e$, which contradicts the nonzero character of $e$. We will use this in the proof of the next proposition, as well as in that of Proposition 3.5.

Proposition 3.2. Let $A$ be a third-power associative absolute valued algebra.

1. If $e \neq 0$ is an idempotent in $A$ and $x \in A$ an element such that it and its square commute with $e$, then

$$
x^{2}-2(e \mid x)_{e, x} e x+\|x\|^{2} e=0 .
$$

2. Two different commuting nonzero idempotents of $A$ generate a subalgebra isometrically isomorphic to $\stackrel{\star}{\mathbb{C}}$.

3. If a given element $x$ of $A$ and its square $x^{2}$ commute with two commuting different nonzero idempotents $e$ and $f$, then $x$ belongs to the subalgebra $\mathbb{R e}+\mathbb{R} f$ generated by $e$ and $f$.

Proof: 1. By third-power associativity, from the commutativity of $e$ and $x$ it follows that of $e$ with $e x$. Now (10) is a particular case of (3).

2. By part 1 , if $e \neq 0 \neq f$ are different commuting idempotents then $e-2(e \mid f)_{e, f} e f+f=0$. Furthermore, $(e \mid f)_{e, f} \neq 0$, because $(e \mid$ $f)_{e, f}=0$, implies $e=-f$, a contradiction. From $(e \mid f)_{e, f} \neq 0$ it follows ef $\in \mathbb{R} e+\mathbb{R} f$, which proves that $\mathbb{R} e+\mathbb{R} f$ is a commutative subalgebra. By Lemma 2.1, $\mathbb{R} e+\mathbb{R} f$ is isometrically isomorphic to $\stackrel{\star}{\mathbb{C}}$.

3. We assume $x \neq 0$. By part 1 ,

$$
x^{2}-2(e \mid x)_{e, x} e x+\|x\|^{2} e=0, \quad x^{2}-2(f \mid x)_{f, x} f x+\|x\|^{2} f=0 .
$$

Subtracting both equalities we obtain $\|x\|^{2}(f-e)=2\left((f \mid x)_{f, x} f-(e \mid\right.$ $\left.x)_{e, x} e\right) x$. In particular, $(f \mid x)_{f, x} f-(e \mid x)_{e, x} e \neq 0$. By part $2, B=$ $\mathbb{R} e+\mathbb{R} f$ is a subalgebra isometrically isomorphic to $\stackrel{\star}{\mathbb{C}}$. In particular there exists some $z \in B$ such that $\|x\|^{2}(f-e)=2\left((f \mid x)_{f, x} f-(e \mid x)_{e, x} e\right) z$. 
So $\left((f \mid x)_{f, x} f-(e \mid x)_{e, x} e\right)(x-z)=0$. Thus $x=z \in B$, because $A$ has not nonzero zero divisors.

Remark 3.3. Part 2 of Proposition 3.2 appears in [5, Lemme 3.12], where a different proof is given.

Let $A$ be a third-power associative absolute valued algebra. We will denote by $\mathcal{E}$ the set of the nonzero idempotents of $A$. If $g \in \mathcal{E}$ and $w$ is an element of $A$ such that $w \notin \mathbb{R} g$, then we will denote by $\mathcal{E}_{w}(g)$ the set of the $e \in \mathcal{E}$ for which there exists some $w^{\prime} \in \mathbb{R} g+\mathbb{R} w$ such that $\left(w^{\prime 2} \mid w^{\prime}\right)_{w^{\prime}, 2}=0$ and $w^{\prime 2}=-e$. In this case we also say that $w^{\prime}$ is a $(g, w)$-square root of $-e$. In the following proposition we provide sufficient conditions assuring that $\mathcal{E}_{w}(g)$ has only one idempotent $e$ different from $g$.

Proposition 3.4. Let $A$ be a third-power associative absolute valued algebra, $g$ a nonzero idempotent of $A$, and $w$ a norm-one element of $A$ satisfying $[w, g] \neq 0$, so that $g$ commutes with $w^{2}$ and with each idempotent of $\mathcal{E}_{w}(g)$. Then $\mathcal{E}_{w}(g)$ consists of only one nonzero idempotent e. Furthermore, $-w^{2}=e \neq g$.

Proof: Assume $e \in \mathcal{E}_{w}(g)$. Then $e$ is a nonzero idempotent and there exists some $w^{\prime}$ in the vector plane $P=\mathbb{R} g+\mathbb{R} w$ satisfying $\left(w^{\prime 2} \mid w^{\prime}\right)_{w^{\prime}, 2}=$ 0 and $w^{\prime 2}=-e$. So $w^{\prime}$ and $e$ commute, $w^{\prime} \neq \pm g$ and $P=\mathbb{R} g+\mathbb{R} w^{\prime}$. This implies $e \neq g$, because if $e=g$ then $\left[g, w^{\prime}\right]=0$ and $g$ would commute with all elements of $P$ and, in particular, $[g, w]=0$ which is a contradiction. Writing $w^{\prime}=\xi g+\eta w(\xi, \eta \in \mathbb{R})$, we have $w^{\prime 2}=$ $\xi^{2} g+\xi \eta(g w+w g)+\eta^{2} w^{2}$. Since $\left[w^{2}, g\right]=0$ and $\left[{w^{\prime}}^{2}, g\right]=0$, we obtain $0=\left[g, w^{\prime 2}\right]=\xi \eta[g, g w+w g]=\xi \eta[g, w]$. Thus, $\xi \eta=0$. The possibility $\eta=0$ is ruled out, because we have shown at the start of this proof that $w^{\prime} \neq \pm g$. So $\xi=0, w^{\prime}= \pm w$ and $-w^{2}=e \neq g$. In particular, $w$ determines completely $e$ and in consequence $\mathcal{E}_{w}(g)=\{e\}$.

Proposition 3.5. Let $A$ be a third-power associative absolute valued algebra, $W$ a vector subspace of $A$, and $g \in W$ a nonzero idempotent commuting with every element of $W$, with the property that for every $w \in W$ such that $w \notin \mathbb{R} g$, the idempotents in the set $\mathcal{E}_{w}(g)$ commute with $\mathrm{g}$. Then the following assertions hold:

1. For every $w \in W$, the norm of $\mathbb{R} g+\mathbb{R} w$ derives from an inner product.

2. $u^{2}=-\|u\|^{2} g$ for all $u \in W$ and orthogonal to $g$.

3. If the elements of $W$ pairwise commute then $\operatorname{dim} W \leq 2$. 
4. If the elements of $W$ pairwise commute and, in addition, for each $w \in W$ and $v \in \mathbb{R} g+\mathbb{R} w+\mathbb{R}(g w)$, with $v \notin \mathbb{R} g$, the idempotent $g$ commutes with each idempotent of $\mathcal{E}_{v}(g)$, then $W$ is a subalgebra of $A$ isometrically isomorphic to $\mathbb{R}, \mathbb{C}$, or $\stackrel{\star}{\mathbb{C}}$.

Proof: Since the elements of $W$ commute with $g$, for every $w \in W$ the norm of $\mathbb{R} g+\mathbb{R} w$ derives from an inner product (see [16, Lemma 1]). This proves part 1. If $\operatorname{dim} W=1$ the remaining assertions of Proposition 3.5 are trivial, because in this case $W=\mathbb{R} g$. So we will assume without loss of generality that $\operatorname{dim} W \geq 2$, and we will proceed in a sequence of steps.

Step 1: For each $w \in W$ with $w \notin \mathbb{R} g$, all $e^{\prime} \in \mathcal{E}_{w}(g)$ with $e^{\prime} \neq g$ and every $(g, w)$-square root $w^{\prime}$ of $-e^{\prime}$, we have that $\mathbb{R} g+\mathbb{R} e^{\prime}$ is a subalgebra isometrically isomorphic to $\stackrel{\star}{\mathbb{C}}$ and, in addition, $\mathbb{R} g+\mathbb{R} e^{\prime}=\mathbb{R} g+\mathbb{R} w^{\prime}=$ $\mathbb{R} g+\mathbb{R} w \subset W$. In particular, if some $\mathcal{E}_{w}(g)$ contains elements different from $g$ then $W$ contains some subalgebra isometrically isomorphic to $\stackrel{\star}{\mathbb{C}}$.

Indeed, from $w^{\prime} \in \mathbb{R} g+\mathbb{R} w \subset W$ it follows that $w^{\prime}$ commutes with $g$. Since $w^{\prime 2}=-e^{\prime}, w^{\prime 2}$ commutes with $g$ and $w^{\prime}$ commutes with $e^{\prime}$. By part 3 of Proposition 3.2, $w^{\prime} \in \mathbb{R} g+\mathbb{R} e^{\prime}$. Now we observe that $w^{\prime} \notin \mathbb{R} g$, because if it were $w^{\prime} \in \mathbb{R} g$ then $w^{\prime}= \pm g,-e^{\prime}=w^{\prime 2}=g^{2}=g$, which is impossible for the nonzero idempotents $e$ and $g$ of $A$. From $w^{\prime} \notin \mathbb{R} g$ and $w^{\prime} \in \mathbb{R} g+\mathbb{R} e^{\prime}$ it follows that $e^{\prime} \in \mathbb{R} g+\mathbb{R} w^{\prime}$. Thus, $\mathbb{R} g+\mathbb{R} e^{\prime}=\mathbb{R} g+\mathbb{R} w^{\prime} \subset$ $W$ and $W$ contains the distinct idempotents $g$ and $e^{\prime}$. By part 2 of Proposition $3.2, \mathbb{R} g+\mathbb{R} e^{\prime}$ is isometrically isomorphic to $\stackrel{\star}{\mathbb{C}}$. Furthermore, from $w^{\prime} \notin \mathbb{R} g$ and $w^{\prime} \in \mathbb{R} g+\mathbb{R} w$ it follows $\mathbb{R} g+\mathbb{R} w^{\prime}=\mathbb{R} g+\mathbb{R} w$.

Step 2: $g \in \mathcal{E}_{w}(g)$ for every $w \in W$ such that $w \notin \mathbb{R} g$.

Indeed, by Proposition 3.1 if $w \in W$ and $w \notin \mathbb{R} g$, then there exists $w^{\prime} \in \mathbb{R} g+\mathbb{R} w$ such that $e^{\prime}=-w^{\prime 2}$ is a nonzero idempotent satisfying $\left(w^{\prime} \mid e^{\prime}\right)_{w^{\prime}, 2}=0$. Without loss of generality we will assume $e^{\prime} \neq g$. By step $1, \mathbb{R} g+\mathbb{R} e^{\prime}=\mathbb{R} g+\mathbb{R} w \subset W$ and $\mathbb{R} g+\mathbb{R} e^{\prime}$ is a subalgebra isometrically isomorphic to $\stackrel{\star}{\mathbb{C}}$. If $v_{0}$ is a norm-one element of $\mathbb{R} g+\mathbb{R} e^{\prime}$ orthogonal to $g$, then $v_{0}^{2}=-g$ and $g \in \mathcal{E}_{w}(g)$.

Step 3: $u^{2}=-\|u\|^{2} g$ for all $u \in W$ and orthogonal to $g$.

By step $2, g \in \mathcal{E}_{u}(g)$. So, it follows the existence of $u^{\prime} \in \mathbb{R} g+\mathbb{R} u$ such that $\left(u^{\prime 2} \mid u^{\prime}\right)_{u^{\prime}, 2}=0$, and $u^{\prime 2}=-g$. So $\left(u^{\prime} \mid g\right)_{u^{\prime}, 2}=0$ and $\mathbb{R} u=\mathbb{R} u^{\prime}$. In consequence, $u= \pm\|u\| u^{\prime}$ and $u^{2}=-\|u\|^{2} g$.

Step 4: If the elements of $W$ pairwise commute then $\operatorname{dim} W=2$. 
Indeed, consider two norm-one elements $u_{1}, u_{2} \in W$ orthogonal to $g$. By step $3, u_{1}^{2}=-g=u_{2}^{2}$ and in consequence $0=\left(u_{1}-u_{2}\right)\left(u_{1}+u_{2}\right)$. Thus, $u_{2}= \pm u_{1}$ which implies $\operatorname{dim} W=2$.

Step 5. If the elements of $W$ pairwise commute and, in addition, for each $w \in W$ and $v \in \mathbb{R} g+\mathbb{R} w+\mathbb{R}(g w)$, with $v \notin \mathbb{R} g$, the idempotent $g$ commutes with each idempotent of $\mathcal{E}_{v}(g)$, then $W$ is a subalgebra isometrically isomorphic to $\mathbb{C}$ or $\stackrel{\star}{\mathbb{C}}$.

Indeed, since $\operatorname{dim} W=2$ there exists a norm one element $u \in W$ which is orthogonal to $g$. By step $3, u^{2}=-g$. By third-power associativity, the elements $g, u$, and $g u$ pairwise commute. Step 4 applied to the vector subspace $\mathbb{R} g+\mathbb{R} u+\mathbb{R}(g u)$ implies $g u \in \mathbb{R} g+\mathbb{R} u$ and consequently the multiplication map $L_{g}$ induces an isometric linear map in the vector subspace $W=\mathbb{R} g+\mathbb{R} u$. Therefore, $g u= \pm u$ and $W$ is a subalgebra isometrically isomorphic to $\mathbb{C}$ or $\stackrel{\star}{\mathbb{C}}$.

Proposition 3.6. Let $A$ be a third-power associative absolute valued algebra, $g \in A$ a nonzero idempotent commuting with all idempotents of $A$, and $C(g)$ the vector subspace of the elements of $A$ commuting with $g$. Then the following assertions hold:

1. If $x \in C(g)$ then

$$
\left(L_{g}+R_{g}\right)^{2} x=4 x, \quad g(g x)=x=(x g) g .
$$

2. The norm of $C(g)$ derives from an inner product, $C(g)$ is a subalgebra of $A^{+}$, and if $V$ is the vector subspace of the elements of $C(g)$ orthogonal to $g$, then $g v= \pm v$ for any $v \in V$ and the commutative symmetrized product $\bullet$ of $C(g)$ is given as in (6) for any $\alpha, \beta \in \mathbb{R}$ and $v, w \in V$.

3. An element $x$ of $C(g)$ commutes with a nonzero idempotent $e \neq g$ if and only if $x \in \mathbb{R} g+\mathbb{R e}$.

4. $\left(L_{g}+R_{g}\right)^{3}-\left(L_{g}+R_{g}\right)^{2}-4\left(L_{g}+R_{g}\right)+4 \mathrm{Id}=0$.

5. A splits as a direct sum of vector subspaces $A=A_{2} \oplus A_{-2} \oplus A_{1}$, where

$$
\begin{aligned}
A_{2} & =\left\{x \in A:\left(L_{g}+R_{g}\right) x=2 x\right\}, \\
A_{-2} & =\left\{x \in A:\left(L_{g}+R_{g}\right) x=-2 x\right\}, \\
A_{1} & =\left\{x \in A:\left(L_{g}+R_{g}\right) x=x\right\} .
\end{aligned}
$$

6. $z^{2} \in C(g)$ for all $z \in A_{1}$.

7. The vector subspace $C(g)$ agrees with $A_{2} \oplus A_{-2}$, furthermore either $A_{-2}=0$ or $A_{2}=\mathbb{R} g$. 
8. $A_{2}$ and $A_{-2}$ are orthogonal subspaces of $C(g)$ and

(11) $A_{2}=\{x \in A: x g=x=g x\}$ and $A_{-2}=\{x \in A: x g=-x=g x\}$.

Proof: 1. By Proposition 3.5, for every $x$ commuting with $g$ the vector subspace $\mathbb{R} g+\mathbb{R} x$ is a subalgebra isometrically isomorphic to $\mathbb{R}, \mathbb{C}$, or $\stackrel{\star}{\mathbb{C}}$. So $g$ is a unit or a para-unit element of this subalgebra and, in particular, relations 1 hold.

2. Since each $x$ of $C(g)$ generates with $g$ a subalgebra isometrically isomorphic to $\mathbb{R}, \mathbb{C}$, or $\stackrel{\star}{\mathbb{C}}$, part 2 is an immediate consequence of Proposition 2.5.

3. The commutativity of $e$ and $g$ obviously implies that the elements of $\mathbb{R} g+\mathbb{R} e$ commute with $e$. On the other hand, if $x=\alpha g+v(\alpha \in$ $\mathbb{R}, v \in V$ ) commutes with $e$ then, from the commutativity of $g$ and $e$, it follows $[v, e]=0$. Using the commutative product $\bullet$ of part 2, we obtain $\left[x^{2}, e\right]=\left[\left(\alpha^{2}-\|v\|^{2}\right) g+2 \alpha g v, e\right]= \pm 2 \alpha[v, e]=0$. But $x^{2}$ also commutes with $g$, because $x^{2} \in \mathbb{R} g+\mathbb{R} v \subset C(g)$. By Proposition 3.2, $x \in \mathbb{R} g+\mathbb{R} e$.

4. By third-power associativity for every $y \in A$ the element $g y+y g-y$ commutes with $g$. By part 1,

$$
\left(L_{g}+R_{g}\right)^{2}\left(L_{g}+R_{g}-\mathrm{Id}\right) y=4\left(L_{g}+R_{g}-\mathrm{Id}\right) y,
$$

which can be rewritten in the way $\left(L_{g}+R_{g}\right)^{3}-\left(L_{g}+R_{g}\right)^{2}-4\left(L_{g}+\right.$ $\left.R_{g}\right)+4 \mathrm{Id}=0$.

5. The polynomial $X^{3}-X^{2}-4 X+4$ has roots $2,-2$, and 1 . So $A$ has the decomposition in vector subspaces claimed in 5 .

6. By linearization of the third-power associativity identity, if $z \in A_{1}$ then $\left[z^{2}, g\right]=-[z g+g z, z]=-[z, z]=0$. Therefore, $z^{2} \in C(g)$.

7. By (8), for every $x \in A_{2}$ we have $[2 x, g]=[g x+x g, g]=-\left[g^{2}, x\right]=$ - $[g, x]$. Thus, $[x, g]=0$ and $A_{2} \subset C(g)$. In a similar way it can be proved the inclusion $A_{-2} \subset C(g)$. Therefore, $A_{2} \oplus A_{-2} \subset C(g)$.

In order to complete the proof of part 7 we consider the vector subspace $V$ of the elements of $C(g)$ which are orthogonal to $g$ and we will assume without loss of generality that $V \neq 0$. Let $v$ be a norm-one element in $V$. By part 2, $g v= \pm v=v g$. Therefore, $v \in A_{2} \cup A_{-2}$ and $V \subset A_{2} \cup A_{-2}$. Since a real vector space cannot be a union of two proper vector subspaces, we obtain $A_{2} \cap V=V$ or $A_{-2} \cap V=V$. So $V \subset A_{2}$ or 
$V \subset A_{-2}$ and this yields to $C(g) \subset A_{2}$ or $C(g) \subset \mathbb{R} g \oplus A_{-2}$. Therefore, $A_{-2}=0$ or $A_{2}=\mathbb{R} g$.

8. By part 7 we obtain (11). Since $L_{g}$ is an isometric linear map (not necessarily onto), for all $x \in A_{2}$ and $u \in A_{-2}$ we have $(x \mid u)=(g x \mid$ $g u)=-(x \mid u)$ and consequently $(x \mid u)=0$.

As a corollary of the above proposition we obtain the following ElMallah's result (see [10, Theorem 3.7] and [9, Theorem 6]).

Corollary 3.7 (M. L. El-Mallah). Let $A$ be a third-power associative absolute valued algebra. If $g \neq 0$ is an idempotent commuting with all the elements of $A$, then $A$ is isometrically isomorphic to $\mathbb{R}, \mathbb{C}, \mathbb{H}, \mathbb{O}, \stackrel{\star}{\mathbb{C}}$, $\stackrel{\star}{\mathbb{H}}$, or $\stackrel{\star}{\mathbb{O}}$.

Proof: By Proposition 3.6, the norm of $A$ derives from an inner product and either $A=\mathbb{R} g \oplus A_{-2}$ or $A=A_{2}$, with $A_{2}$ and $A_{-2}$ the vector subspaces given as in (11). If $A=A_{2}$ then $A$ has $g$ as a unit element, and as a consequence of the generalized Hurwitz Theorem, we get $A$ isometrically isomorphic to $\mathbb{R}, \mathbb{C}, \mathbb{H}$, or $\mathbb{O}$. In order to complete the proof we now consider the case that $A=\mathbb{R} g \oplus A_{-2}$ with $A_{-2} \neq 0$. Now $L_{g}\left(=R_{g}\right)$ is the unique linear map fixing $g$ and mapping every element of its orthogonal vector subspace to its opposite. By changing the product of $A$ by the new product $\circ$ given by $x \circ y=(g x)(y g)$, we obtain a new algebra $(A, \circ)$ having $g$ as a unit element and being an absolute valued algebra respect to the same norm that $A$. Taking into account that $\operatorname{dim} A \geq 2$, now the generalized Hurwitz Theorem gives $(A, \circ)$ isometrically isomorphic to $\mathbb{C}, \mathbb{H}$, or $\mathbb{O}$. Since $x y=\left(L_{g}(x)\right) \circ$ $\left(R_{g}(y)\right)$ we obtain that if $A=\mathbb{R} g \oplus A_{-2}$ with $A_{-2} \neq 0$ then $A$ is isometrically isomorphic to $\stackrel{\star}{\mathbb{C}}, \stackrel{\star}{\mathbb{H}}$, or $\stackrel{\star}{\mathbb{O}}$.

Remark 3.8. In [7, Theorem 4.6] we have obtained the same conclusion that in the above corollary even in the case that the third-power associative condition is substituted by the weaker assumption that the algebraic identity $\left(x^{2} x^{2}\right) x^{2}=x^{2}\left(x^{2} x^{2}\right)$ holds in $A$.

\section{The main theorem}

Obviously $\mathbb{R}, \mathbb{C}, \mathbb{H}, \mathbb{O}, \stackrel{\star}{\mathbb{C}}, \stackrel{\star}{\mathbb{H}}$, and $\stackrel{\star}{\mathbb{O}}$ are third-power associative absolute valued algebras with one idempotent commuting with all the idempotents. The next theorem, which is the main theorem of this paper, shows that these algebras are the unique absolute valued algebras satisfying these conditions. 
Theorem 4.1. A third-power associative absolute valued algebra $A$ has a nonzero idempotent commuting with the remaining idempotents if and only if $A$ is isometrically isomorphic to $\mathbb{R}, \mathbb{C}, \mathbb{H}, \mathbb{O}, \stackrel{\star}{\mathbb{C}}, \stackrel{\star}{\mathbb{H}}$, or $\stackrel{\star}{\mathbb{O}}$.

Proof: As we have pointed out $\mathbb{R}, \mathbb{C}, \mathbb{H}, \mathbb{O}, \stackrel{\star}{\mathbb{C}}, \stackrel{\star}{\mathbb{H}}$, and $\stackrel{\star}{\mathbb{O}}$ satisfy the required conditions.

Now we assume a third-power associative algebra $A$ having a nonzero idempotent $g$ commuting with all idempotents of $A$. By Proposition 3.6 and Corollary 3.7, it suffices to show that the vector subspace $A_{1}=\{x \in$ $\left.A:\left(L_{g}+R_{g}\right) x=x\right\}$ is zero. We will prove this by contradiction. So we will assume the existence of some norm-one element $z$ in $A_{1}$. By Proposition 3.6, $[z, g] \neq 0$ and $\left[z^{2}, g\right]=0$. By Proposition 3.4, there exists a nonzero idempotent $e$ orthogonal to $z$ such that

$$
-z^{2}=e \neq g \text {. }
$$

By Proposition 3.1, there exist $\alpha, \delta \in \mathbb{R}$ such that $-(\alpha g+\delta(e+z))^{2}$ is a nonzero idempotent. In particular, $\delta \neq 0$ and $(\alpha g+\delta(e+z))^{2} \in C(g)$. Furthermore, using (12) we obtain

$$
\begin{aligned}
(\alpha g+\delta(e+z))^{2} & =\alpha^{2} g+\delta^{2}\left(e+z^{2}+2 e z\right)+\alpha \delta g(e+z)+\alpha \delta(e+z) g \\
& =\alpha^{2} g+2 \delta^{2} e z+\alpha \delta(g e+e g)+\alpha \delta(g z+z g) \\
& =\alpha^{2} g+2 \alpha \delta g e+\alpha \delta z+2 \delta^{2} e z
\end{aligned}
$$

which implies that $e z \in C(g)+\mathbb{R} z$. Writing $e z=b+\rho z$ with $b \in C(g)$ and $\rho \in \mathbb{R}$, we see that $[b, e]=[e z, e]-\rho[z, e]=0$. So, by Proposition 3.6, $b \in \mathbb{R} g+\mathbb{R} e$ and $e z \in \mathbb{R} g+\mathbb{R} e+\mathbb{R} z$.

Now we assert that $e z \in \mathbb{R} e+\mathbb{R} z$. Indeed, in the contrary case $\mathbb{R} e z+$ $\mathbb{R} e+\mathbb{R} z=\mathbb{R} g+\mathbb{R} e+\mathbb{R} z$. The pairwise commutativity of the elements of $\mathbb{R} e z+\mathbb{R} e+\mathbb{R} z$ yields, in particular, to $[g, z]=0$. This contradiction proves that $e z \in \mathbb{R} e+\mathbb{R} z$. So the restriction of the linear map $L_{e}$ can be seen as an isometric linear map of the euclidean plane $\mathbb{R} e+\mathbb{R} z$. By orthogonality of $e$ and $z$ it follows that $e z= \pm z$. Therefore, $\mathbb{R} e+\mathbb{R} z$ is a subalgebra of $A$ isometrically isomorphic to $\mathbb{C}$ or $\stackrel{\star}{\mathbb{C}}$. Finally we conclude the proof observing that both possibilities are impossible.

The case that $\mathbb{R} e+\mathbb{R} z$ is isometrically isomorphic to $\stackrel{\star}{\mathbb{C}}$ is ruled out from the fact that under this assumption $-\frac{1}{2} e+\frac{\sqrt{3}}{2} z$ would be a nonzero idempotent which does not belong to $C(g)$. Now we will assume $\mathbb{R} e+\mathbb{R} z$ isometrically isomorphic to $\mathbb{C}$. So $e z=z$. Since $\mathbb{R} e+\mathbb{R} g$ is a subalgebra isometrically isomorphic to $\stackrel{\star}{\mathbb{C}}$, we can assure the existence of some $v_{0} \in$ 
$\mathbb{R} g+\mathbb{R} e \subset C(g)$ satisfying $v_{0}^{2}=-e, e v_{0}=-v_{0}$, and $g=-\frac{1}{2} e+\frac{\sqrt{3}}{2} v_{0}$. So $z=g z+z g=-\frac{1}{2}(e z+z e)+\frac{\sqrt{3}}{2}\left(v_{0} z+z v_{0}\right)=-z+\frac{\sqrt{3}}{2}\left(v_{0} z+z v_{0}\right)$ and consequently $v_{0} z+z v_{0}=\frac{4}{\sqrt{3}} z$. Let $\lambda$ and $\mu$ be arbitrary real numbers. Then

$$
\left(\lambda z+\mu v_{0}\right)^{2}=\lambda^{2} z^{2}+\mu^{2} v_{0}^{2}+\lambda \mu\left(z v_{0}+v_{0} z\right)=-\left(\lambda^{2}+\mu^{2}\right) e+\frac{4}{\sqrt{3}} \lambda \mu z .
$$

On the other hand, for every pair of elements $\gamma e+\lambda z+\mu v_{0}$ and $\gamma^{\prime} e+$ $\lambda^{\prime} z+\mu^{\prime} v_{0}$ of $\mathbb{R} e+\mathbb{R} z+\mathbb{R} v_{0}$ we have

$$
\left[\gamma e+\lambda z+\mu v_{0}, \gamma^{\prime} e+\lambda^{\prime} z+\mu^{\prime} v_{0}\right]=\left(\lambda \mu^{\prime}-\mu \lambda^{\prime}\right)\left[z, v_{0}\right]
$$

From (13) and (14) it follows

$$
\left[\lambda z+\mu v_{0},\left(\lambda z+\mu v_{0}\right)^{2}\right]=-\frac{4}{\sqrt{3}} \lambda \mu^{2}\left[z, v_{0}\right] .
$$

We have $\left[z, v_{0}\right] \neq 0$, because $\left[z, v_{0}\right]=0$ implies $[z, g]=0$. From (15) we obtain that the commutativity of $\lambda z+\mu v_{0}$ with $\left(\lambda z+\mu v_{0}\right)^{2}$ is equivalent to the condition $\lambda \mu^{2}=0$, which contradicts the third-power associativity of $A$. This contradiction completes the proof.

Remark 4.2 (On the Urbanik and Wright Theorems). We can observe that the arguments given in this paper do not depend neither on the commutative nor on the noncommutative Urbanik and Wright Theorem. The original proof of this last theorem given in [16] has a crucial step which is devoted to show that in each unital absolute valued algebra $A$ the unital subalgebra $A_{x}$ generated by any nonzero element $x$ is isomorphic to $\mathbb{R}$ or $\mathbb{C}$. This implies the third-power associativity of $A$. Now Theorem 4.1 can be considered in order to obtain as an immediate consequence that $A$ is isometrically isomorphic to $\mathbb{R}, \mathbb{C}, \mathbb{H}$, or $\mathbb{O}$ (in the original proof the same conclusion is obtained by invoking the Albert theorem on the determination of the absolute valued algebraic algebras with a unit element [2]). Since Proposition 3.1 implies the existence of nonzero idempotents in any commutative absolute valued algebra, it is also possible to obtain the commutative Urbanik and Wright Theorem directly as a particular case of Theorem 4.1 (even it can be derived from Corollary 3.7).

Another extension of the commutative Urbanik and Wright Theorem is given in [8, Corollary 1.6], where it is proved that if $A$ is a real commutative algebra without nonzero zero divisors which is a prehilbert space whose norm satisfies $\left\|a^{2}\right\|=\|a\|^{2}$ for every $a \in A$ and if, in addition, $A$ has a norm-one idempotent $e$ such that $\|e a\|=\|a\|$ for any $a \in A$, then $A$ is isomorphic to $\mathbb{R}, \mathbb{C}$, or $\stackrel{\star}{\mathbb{C}}$. 
The following corollary is a particular case of Theorem 4.1.

Corollary 4.3 (A. Chandid). Let A be a third-power associative absolute valued algebra. Then the nonzero idempotents of $A$ commute pairwise if and only if $A$ is isometrically isomorphic to $\mathbb{R}, \mathbb{C}, \mathbb{H}, \mathbb{O}$, or $\stackrel{\star}{\mathbb{C}}$. In particular, $A$ has only one nonzero idempotent if and only if $A$ is isometrically isomorphic to $\mathbb{R}, \mathbb{C}, \mathbb{H}$, or $\mathbb{O}$.

Remark 4.4. Our main theorem also includes the characterization of the power-associative absolute valued algebras given by M. L. El-Mallah and A. Micali [11]. Indeed, if $A$ is a power-associative absolute valued algebra then $A$ has some nonzero idempotent $e$. This can be obtained either form Proposition 3.1 (see also the previous comments to it) or as a consequence of the commutative Urbanik and Wright Theorem. Now as in [15, p. 134] we obtain that $e$ is the only nonzero idempotent of $A$, and even that $e$ is unit element of $A$.

\section{Acknowledgements}

The author wishes to express his gratitude to Professor Miguel Cabrera García for his valuable comments and for improving the proof of Proposition 3.5.

\section{References}

[1] A. A. Albert, Absolute valued real algebras, Ann. of Math. (2) 48 (1947), 495-501.

[2] A. A. Albert, Absolute-valued algebraic algebras, Bull. Amer. Math. Soc. 55 (1949), 763-768. DOI: 10.1090/S0002-9904-194909278-9.

[3] A. A. Albert, A note of correction, Bull. Amer. Math. Soc. 55 (1949), p. 1191. DOI: 10.1090/S0002-9904-1949-09358-8.

[4] M. Cabrera García and A. Rodríguez Palacios, "Non-associative normed algebras. Vol 1: The Vidav-Palmer and GelfandNaimark theorems", book in progress, Cambridge University Press, 2014.

[5] A. Chandid, Algèbres absolument valuées qui satisfont à $\left(x^{p}, x^{q}, x^{r}\right)=0, \mathrm{PhD}$ thesis, Faculté des Sciences, Université Hassan II, Casablanca (2009).

[6] J. A. Cuenca Mira, On one-sided division infinite-dimensional normed real algebras, Publ. Mat. 36(2A) (1992), 485-488. DOI: 10.5565/PUBLMAT_362A92_14. 
[7] J. A. Cuenca Mira, On composition and absolute-valued algebras, Proc. Roy. Soc. Edinburgh Sect. A 136(4) (2006), 717-731. DOI: $10.1017 / \mathrm{S} 0308210500004686$.

[8] J. A. Cuenca Mira, Some classes of pre-Hilbert algebras with norm-one central idempotent, Israel J. Math. 193(1) (2013), 343-358. DOI : 10.1007/s11856-012-0069-1.

[9] M. L. El-Mallah, Absolute valued algebras with an involution, Arch. Math. (Basel) 51(1) (1988), 39-49. DOI: 10.1007/BF01194152.

[10] M. L. El-Mallah, Absolute valued algebras containing a central idempotent, J. Algebra 128(1) (1990), 180-187. DOI: 10.1016/ 0021-8693 (90) 90048-S.

[11] M. L. El-Mallah And A. Micali, Sur les algèbres normées sans diviseurs topologiques de zéro, Bol. Soc. Mat. Mexicana (2) 25(1) (1980), 23-28.

[12] S. OKubo, Dimension and classification of general composition algebras, in: "Proceedings of the Third Workshop on Lie-Admissible Formulations" (Univ. Massachusetts, Boston, Mass., 1980), Part A, Hadronic J. 4(2) (1980/81), 216-273.

[13] A. Rodríguez-Palacios, One-sided division absolute valued algebras, Publ. Mat. 36(2B) (1992), 925-954. DOI : 10.5565/PUBLMAT_ 362B92_12.

[14] A. Rodríguez Palacios, Absolute-valued algebras, and absolutevaluable Banach spaces, in: "Advanced courses of mathematical analysis I", World Sci. Publ., Hackensack, NJ, 2004, pp. 99-155. DOI: $10.1142 / 9789812702371_{-} 0005$.

[15] R. D. SchAFER, "An introduction to nonassociative algebras", Pure and Applied Mathematics 22, Academic Press, New York-London, 1966.

[16] K. Urbanik and F. B. Wright, Absolute-valued algebras, Proc. Amer. Math. Soc. 11 (1960), 861-866. DOI: 10.1090/S0002-99391960-0120264-6.

Departamento de Álgebra, Geometría y Topología

Facultad de Ciencias

Universidad de Málaga

29071 Málaga

Spain

E-mail address: cuenra@agt.cie.uma.es

Primera versió rebuda el 30 de maig de 2013, darrera versió rebuda el 12 de setembre de 2013. 